\title{
Causality and Advanced Models in Trip Mode Prediction: Interest in Choosing Swissmetro
}

\author{
Huy Pham \\ Department of Civil and Environmental Engineering \\ Xuan Jiang \\ Department of Civil and Environmental Engineering
}

Cong Zhang

Department of Industrial Engineering and Operations Research

\author{
HGP@BERKELEY.EDU \\ J503440616@BERKELEY.EDU
}

OSCAR.ZHANG@BERKELEY.EDU

\begin{abstract}
In March 1998, a survey was conducted on trains running in Switzerland in order to gauge the public interest in utilizing a new mag-lev train system operated by Swissmetro. The survey results are often used as data in order to facilitate trip mode prediction models. In order to explore the double descent hypothesis, which posits that overparameterization of models helps generalization rather than harming it, a multinomial logit model with regularization is compared against neural-network models that overparameterized in this study. We finally achieved $67 \%$ accuracy on the neural-network compared with the $44 \%$ accuracy of the multinomial logit model. The models are tested against a common testing model, and the accuracy performance of the models are examined. Additionally, to further investigate the choice of parameters commonly used in prediction models, several causal inference studies are carried out to adjust the effect of the survey location (as a proxy for existing mode choice) and travelers' high-income status against possible confounders. Ultimately, causal inference further strengthened the variables survey location and traveler income as effective predictors in models, especially when treated for causal variables.
\end{abstract}

\section{Introduction}

In transportation area, researchers have done things regarding arterial (Bauranov et al., 2021), electrical vehicles (Everts and Jiang, 2021) and individual behaviors

For the purposes of predicting travel demands, the research community have made many efforts and iterations of mode choice models. It is no surprise that distance is a very important variable in many models, and research (Hagenauer and Helbich, 2017) have been conducted on other variables in combination with distance using traditional machine learning methods such as support vector machine, naive neural network, among many others. However, there is still a relatively small amount research done on causal inference between different variables in mode choice models. 
There are some causal inference study in travel demand modeling based on the hypothesis of the reasons for travel demands, but they don not solve the problem of drawing causal inference from the travel demand model (Brathwaite and Walker, 2018).

Additionally, though artificial neural nets have been used in the past as a mode choice model (Omrani, 2015) (Celikoglu, 2006), many models still consider forms of regularization in order to perform well in generalizing on unseen data. Therefore, this project seeks to partially compare and explain the benefits and tradeoff between regularization and generalization between models that restrict variables used against models that overparameterize. We seek to explain and explore the possibility that the double descent hypothesis is in play: does overparameterization of models helps rather than harm generalization?

To that end, the Swissmetro dataset will be analyzed under two sets of models to compare regularization's performance with regards to generalization, as well as possible causal relationships that may arise among the variables. We only study variables that are truly representative of demographics. Most variables are generated randomly for the study and are independent without causal relationships.

\section{Dataset}

The Swissmetro dataset consists of a series of survey responses collected from travelers between major urban centers in Switzerland (Bierlaire et al., 2001). Surveys were collected in two major batches: a set where surveys were collected from respondents traveling on a train, and a set where surveys were mailed to travelers recorded driving between destinations. The survey contained a series of hypothetical traveling scenarios with variables such as the posited trip's purpose, distance, fare cost, and respondents were asked to choose whether they would drive, take the train, or take the new Swissmetro. Additionally, several demographical pieces of information are collected in the survey. A full list of the variables is available in the technical report of the dataset, (Antonini et al., 2007).

For the purposes of causal inference, we only study variables that are truly representative of demographics. Most variables collected from survey responses are randomly generated scenarios for the study and are independent without causal relationships. Therefore, a subset of true demographic variables (later explained) will be used for causal studies in this project.

\section{Methodology}

\section{A Utility Models}

Our explanatory variables include travel time (alternative specific, as the time experience in each mode is different), and cost (generic across the alternatives, as money generally is). Also included is the headway for Swiss metro and the train, kept separate as there could be differences in waiting. Income to fare ratio is used, which simply divides the travel cost by the individual's income category (plus 1, to avoid dividing by zero), which is used as a measure of how expensive the trip is relative to how much the decision-maker makes. GA ownership indicates whether the decision-maker has the Swiss public transit yearly membership, and first class indicates if the ticket purchased was first class or not. This 
was separated by Metro and train, since these first class experiences may not be equivalent.

Below we present the systematic utility functions of the three alternatives.

$$
\begin{aligned}
V_{\text {Train }}= & A S C_{\text {Train }}+\beta_{\text {TrainTravel Time }} * \text { TravelTimeHours }+\beta_{\text {TrainHeadway }} * \text { Headway Train } \\
& \beta_{\text {TravelCost }} * \text { TravelCost Train }+\beta_{\text {IncomToFareRatio Tain }} * \text { IncomeToFareRatio }+ \\
& \beta_{\text {luggage Truin }} * \text { Luggage Train }+\beta_{\text {GACard }} * \text { GA Dummy }+\beta_{\text {FirstClass Tain }} * \text { FirstClass } \\
V_{\text {Metro }}= & A S C_{\text {Metro }}+\beta_{\text {MetroTravel Time }} * \text { TravelTimeHours }+\beta_{\text {MetroHeadway }} * \text { Headway Metro }+ \\
& \beta_{\text {Travelcost }} * \text { TravelCost Metro }+\beta_{\text {IncomToFareRatio Metro }} * \text { IncomeToFareRatio } \\
& \beta_{\text {luggage }} * \text { Letro } * \text { Luggage Metro }+\beta_{\text {GACard }} * \text { GA Dummy }+\beta_{\text {FirstClass Metro }} * \text { FirstClass } \\
V_{\text {Car }}= & \beta_{\text {CartravelTime }} * \text { TravelTimeHours }+\beta_{\text {TravelCost }} * \text { TravelCost } \\
& \beta_{\text {IncomToFareRatio Car }} * \text { IncomeToFareRatio }
\end{aligned}
$$

\section{B Causal inference}

Although a majority of the variables collected in the Swissmetro dataset are from hypothetical situations from which survey respondents select their preferred mode choice, several demographics and survey status information are also collected that could be used to make inference on trip choice. These include

- LOC: The location where the survey response was collected: either from a train passenger or a car passenger

- AGE \& SEX: The respondent's age and gender

- CAR: The respondent's car ownership

- INC: The respondent's annual income, binned into 4 groups (0-4)

We further simplify the data by grouping respondents who identified within the highest income bracket as high-income earners. Again, we will use the respondent's choice to select Swissmetro for a trip (SM) as the outcome variable.

It is possible for us to use the location of the survey respondent as a proxy variable to predict SM, the respondent's willingness to try the new Swissmetro system. Respondents who return a survey while in a train are likely to be more familiar with public transportation systems and could possibly be more inclined to use a new transit network to travel. Using survey location as our treatment variable, we seek to explore whether or not this relationship is further exacerbated or mitigated when remaining demographic variables are accounted along with survey location.

\section{B.1 Causal diagram}

To facilitate the causal inference study, we propose the following causal network diagram. 


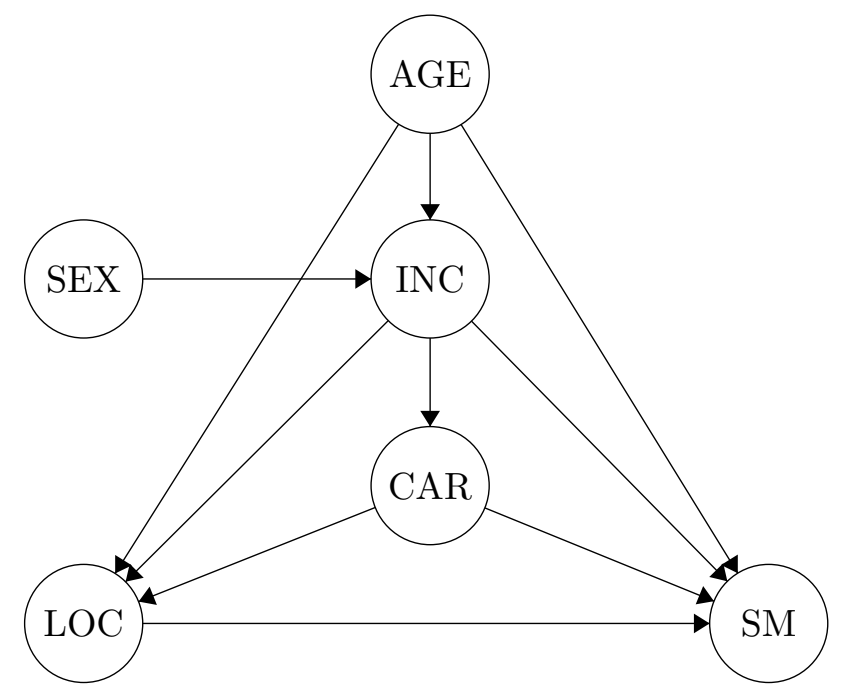

Recall that we posit that survey location is a proxy variable for an individual's existing inclination to use rail transit, such as conventional train or the new Swissmetro. We hypothesize that subject age has a confounding effect on the survey location, income, and the choice of using Swissmetro. Older individuals are likely to have higher income due to longer career experience, and they could possibly be less inclined to use any mode of rail transit for safety and mobility issues. Income correspondingly could confound the survey location, Swissmetro choice, and car ownership. Higher income individuals are more likely to own cars, thus affecting their desire to use rail transit modes. The confounding relationship between car ownership, survey location, and Swissmetro choice is nested in the previous explanation.

Lastly, we include sex as a potential instrumental variable to the outcome variable when treating for high income respondents. Correlation graphs (remember to include correlation plot) show that in this study, male travelers are likely to have higher income; however, we lack a definitive causal relationship between the traveler's sex and their inclination to use Swissmetro. Thus, the instrumental variable may be seen in correlation with the outcome variable but only through an intermediate variable.

\section{B.2 Baseline Average Treatment Effect}

Without controlling for any confounding or instrumental variables, the estimation for the average treatment effect (ATE) of a binary treatment variable $X$ on outcome variable $Y$ is

$$
\begin{aligned}
A T E & =\operatorname{pr}[Y=y \mid X=1]-\operatorname{pr}[Y=y \mid X=0] \\
& =\frac{1}{n_{1}} \sum_{i=1}^{n_{1}} y_{1 i}-\frac{1}{n_{0}} \sum_{i=1}^{n_{0}} y_{0 i}
\end{aligned}
$$

The estimation uses the sample mean of the (binary) outcome variable when subsetting the cases where the treatment is active minus the sample mean outcome when the treatment is withheld. 


\section{B.3 BACKDOOR METHODS}

\section{Adjustment estimation}

A treatment we could use to consider the backdoor effects is the adjustment estimator. Through conditioning of the parent confounding nodes, the causal relationship could be expressed as

$$
\operatorname{pr}[Y=y \mid \operatorname{do}(X:=x)]=\sum_{z} \operatorname{pr}[Y=y \mid X=x, Z=z] \operatorname{pr}[Z=z]
$$

In other words, if we denote $Z=z$ as the event that a traveler belongs to the group $\mathrm{AGE}=a, \mathrm{CAR}=c, \mathrm{INC}=i, \mathrm{SEX}=s$ for all confounding variables, then the adjusted causal effect that the treatment variable has is the average treatment effect within each of the subgroups weighted by the frequency of that combination of variables appearing.

To facilitate the calculation of the conditional probabilities in DoWhy, we carry an assumption of unconfoundedness: that if there exists a variable $U \rightarrow X$ and $U \rightarrow Y$, then we have $\operatorname{pr}(Y \mid X=x, Z=z, U=u)=\operatorname{pr}(Y \mid X=x, Z=z)$.

\section{REGRESSION-BASED METHODS}

Additionally, if we admit a (strong) assumption that all relationships between the treatment variable and the confounders towards the outcome variable are linear, we could fit a regression model to estimate the outcome using the treatment and confounder. For the purposes of this binary treatment study, we utilize the logistic regression.

$$
\operatorname{logit}(y)=\beta_{0}+\beta_{1} x+\sum_{j=2}^{n_{z}} \beta_{j} z
$$

Since the treatment variable is univariate and binary, we report the treatment effect as the coefficient of the treatment variable $x$.

\section{INVERSE PROPENSITY SCORE WEIGHTING (IPW)}

Given the traveler demographics $Z=z$ as described earlier and the treatment variable $X=x$, we express the probability that the individual belonging to that demographic answered the survey on a train as the propensity score $e(z)=\operatorname{pr}(X=1 \mid Z=z)$. From here, we use a logistic regression to estimate the propensity score.

$$
\operatorname{logit}(e(z))=\operatorname{logit}(\operatorname{pr}(X=1 \mid Z=z))=\sum_{i=1}^{d} \beta_{i} z_{i}
$$

Once we have the propensity scores of each category, we can now use the inverse propensity score weighting function to estimate the conditional treatment effect as

$$
A T E=E\left[y \times\left(\frac{x-e(z)}{e(z)(1-e(z))}\right)\right]
$$


Double MACHINE LEARNING (DML)

With a stronger assumption that both the outcome variable and the treatment variable follows a linear model, a double machine learning based method can be used to apply linear regression to estimate causal effects. In this study, both the outcome variable and the treatment variable are regressed using a gradient boosted regressor. Lastly, the final model is fit using a Lasso regression with cross-validation. The double machine learning method is implemented using the EconML package.

\section{B.4 Instrumental VARIABLES}

For certain studies, we seek to estimate the effect of the treatment while considering the correlation with an instrumental variable $V$. This variable is excluded from the outcome variable, meaning that if the causal relationship between $V$ and $X$ were to be removed, there would be no causal relationship between $V$ and $Y$. Further more, the instrumental variable carries an as-if-random assumption that if other variables $U$ affect the outcome variable $Y$, there should be no causal path between $U$ to $V$.

Taking all of the assumptions above is true, we can estimate for the effect of $X$ on $Y$. For a binary univariate treatment, the average treatment effect is equal to the coefficient of the regression

$$
A T E=\beta_{I V}=\left(V^{T} X\right)^{-1} V^{T} y
$$

\section{B.5 Refutations}

To further validate the causal models, we can carry out sensitivity analyses to our causal estimates. One way of doing so is to add an irrelevant additional confounder. On top of the existing causal model as defined by the diagram, a noisy variable is added as a confounder between the treatment and the outcome. We can then observe whether or not the model is invariant to small changes that comes with additional confounders. If the model is robust, we expect that the estimate for the treatment effect should not change significantly.

Additionally, a placebo treatment can be carried out, in which we replace the treatment variable with a randomly generated placebo and reshuffled into the data. In the DoWhy implementation, the treatment variables are simply shuffled among the existing dataset so that its causal relationship is disrupted. In a robust model, the average treatment effect is expected to be mitigated to zero, since the treatment would simply be a useless indicator of effect.

\section{B.6 ImPlementation}

For this project, all causal inference are implemented using the DoWhy package in Python implemented by Microsoft. To further validate the result, several basic implementations of backdoor adjustment estimations and propensity score weighting are manually checked.

\section{Overparameterized Model}

Statistical models are a superfical descrition of reality as they are only required the data associations. For a series of given input feature $X$, we only interested in approximating 
$P(Y \mid X)$ where $Y$ are the target labels. However, without the directional causal link, we may not know whether the change of some certain feature would truly affect the result. In this part, we consider how features without direct causal link affect the traditional statistical model.

Deep networks combines feature learning with classifier training. Our analysis relies on the following observation: over-parameterization and random initialization. We consider a two-layer neural network of the following form.

$$
f(\mathbf{W}, \mathbf{a}, \mathbf{x})=\frac{1}{\sqrt{m}} \sum_{r=1}^{m} a_{r} \sigma\left(\mathbf{w}_{r}^{T} \mathbf{x}\right)
$$

where $\mathbf{x} \in R^{d}$ is the input with random feature, $\mathbf{w}_{r} \in R^{d}$ is the weight vector of the first layer, $a_{r} \in R$ is the output weight and $\sigma(\cdot)$ is the Sigmoid activation function.

\section{Results and Discussion}

\section{A Utility Model and Over Parameterized Model Comparison}

\section{A.1 Utility Model Results}

After implementing Maximum Likelihood Estimation, we got the following Regression Results for Multinomial Logit Model:

\begin{tabular}{|l|l|l|l|}
\hline Dep. Variable: & CHOICE & No. Observations: & 9,810 \\
\hline Model: & Multinomial Logit Model & Df Residuals: & 9,796 \\
\hline Method: & MLE & Df Model: & 14 \\
\hline Date: & Tue, 14 Dec 2021 & Pseudo R-squ.: & 0.277 \\
\hline Time: & $09: 07: 01$ & Pseudo R-bar-squ.: & 0.276 \\
\hline AIC: & $14,814.185$ & Log-Likelihood: & $-7,393.093$ \\
\hline BIC: & $14,914.861$ & LL-Null: & $-10,230.009$ \\
\hline
\end{tabular}

Our a priori expectations were that travel time as an alternative specific variable would be negative for all alternatives, and that cost as a generic variable would generally move people towards taking a train. Having a high income would be positively correlated with driving a car and taking the metro relative to taking the train since we would assume the train is an inferior good like most public transit. Ownership of the GA card would be expected to be positively associated with taking the train or metro, whereas more than one piece of luggage may encourage people to drive. Generally the model matched our expectations, however there are certain exceptions. The biggest is that luggage did not appear to be statistically significant in our final model. Besides this, we were also surprised by how much more greatly high income individuals seemed to prefer the Metro to driving. And we also caught some heterogeneity in our model that gender and income commonly affect decision making. We capture the heterogeneity in income when it comes to fare and found it was more likely for people to choose metro instead of train if their income is higher. Owning the GA card was also a strong point of heterogeneity.

For further choosing the best model for regression, we used various statistical tests in determining the usefulness of our model. Primarily we used t-tests for each parameter to 


\begin{tabular}{|l|l|l|l|l|l|l|}
\hline & coef & std err & $\mathrm{z}$ & $P>|z|$ & {$[0.025$} & $0.975]$ \\
\hline ASC Train & -0.2149 & 0.325 & -0.661 & 0.508 & -0.852 & 0.422 \\
\hline ASC Swissmetro & -0.0336 & 0.275 & -0.122 & 0.903 & -0.573 & 0.506 \\
\hline Travel Time, units:hrs (Train) & -0.9063 & 0.040 & -22.617 & 0.000 & -0.985 & -0.828 \\
\hline Travel Time, units:hrs (Metro) & -0.8652 & 0.039 & -22.203 & 0.000 & -0.942 & -0.789 \\
\hline Travel Time, units:hrs (Car) & -0.7284 & 0.033 & -22.267 & 0.000 & -0.792 & -0.664 \\
\hline Headway, units:hrs, (Train) & -0.3958 & 0.057 & -7.000 & 0.000 & -0.507 & -0.285 \\
\hline Headway, units:hrs, (Swissmetro) & -0.4468 & 0.163 & -2.748 & 0.006 & -0.765 & -0.128 \\
\hline Travel Cost, units: \$1/100 & -0.0906 & 0.006 & -14.542 & 0.000 & -0.103 & -0.078 \\
\hline incomeToFareRatio(Train) & 0.1414 & 0.011 & 12.795 & 0.000 & 0.120 & 0.163 \\
\hline incomeToFareRatio(Metro) & 0.2210 & 0.013 & 16.864 & 0.000 & 0.195 & 0.247 \\
\hline incomeToFareRatio(Car) & 0.0749 & 0.012 & 6.263 & 0.000 & 0.051 & 0.098 \\
\hline Luggage Dummy (Train) & -0.4431 & 0.296 & -1.496 & 0.135 & -1.023 & 0.137 \\
\hline Luggage Dummy (Metro) & -0.3284 & 0.253 & -1.297 & 0.195 & -0.825 & 0.168 \\
\hline GA Card Ownership & 7.3355 & 0.337 & 21.770 & 0.000 & 6.675 & 7.996 \\
\hline
\end{tabular}

evaluate the statistical significance at the $95 \%$ confidence level, for example, the t-test for travel cost equal to $\frac{\widehat{\beta}}{s e_{\beta}}=-0.0931 / 0.006=15.52$ which passes the t-test.

We also do the Likelihood ratio test to test the null hypothesis that all the parameters are zero. When the travel time becomes generic then the log-likelihood ratio test for travel time is $-2\left\{L(\widehat{\beta}\right.$ generic $\left.)-L\left(\widehat{\beta}_{\text {specific }}\right)\right\}=-2(-7357.25-(-7348))=18.5$. Since $\chi^{2}=$ $5.991<18.5$ we can reject the null hypothesis at a $5 \%$ level of significance, meaning the unrestricted (alternative-specific) model is preferred.

Similarly, when the travel cost becomes alternative specific the log-likelihood ratio test for travel cost is as follows: $-2\{L(\widehat{\beta}$ generic $)-L(\widehat{\beta}$ specific $)\}=-2(-7341.9-(-7348))=$ -12.2 Since $\chi^{2}=5.991>-12.2$ we cannot reject the null hypothesis at a $5 \%$ level of significance, meaning the restricted (generic) model is preferred. So far we got our final model through Maximum Likelihood Estimation Regression

\section{A.2 Over-Parameterized Model}

In this part, we test how the random features effect the travel mode classification. The feature we select contains

'mode_id', 'GROUP', 'SURVEY', 'SP', 'PURPOSE', 'FIRST', 'TICKET', 'WHO', 'LUGGAGE', 'AGE',

'MALE', 'INCOME', 'GA', 'ORIGIN', 'DEST',

'travel_time', 'travel_cost', 'headway', 'seat_configuration'

According to the causal graph, the main contribution to the travel mode are age, sex, income, travel time and travel cost. Add irrelavent features will not significant improvement.

To test our hypothesis, we use 10 fold stratified shuffle split trick to preserve the precentage of samples for each class. The final accuracy of the two-layer neural network is around $67 \%$ which is better compared with the hand fined utility model's $44 \%$ accuracy. 


\section{A.3 Comparison of Two Models}

Compared with regularized model with accuracy of $44 \%$, the overparameterized model increases the prediction accuracy to some extent. By adjusting the number of random features and kernel size, the neural network continue to fit the data with low training loss. However, the model are more sensitive to real data as we increase the noise level.

\section{B Causal inference}

The usage of survey location is further investigated with causal methods. Table 1 shows the conditioned average treatment effect (CATE) of survey location as a treatment variable on the likelihood of a traveler choosing Swissmetro for future trips. We provide the baseline ATE as a reference for changes with the causal inference studies, and the returned value has a standard error of 0.019. The basic adjustment formula carried out for survey location's effect conditioned on the confounders shown in Section B.1 returned an effect of 0.108. All placebo treatment studies are simulated 100 times for robustness.

Table 1: Effect of survey location on Swissmetro choice

\begin{tabular}{|c|c|c|c|c|}
\hline Method & CATE & Placebo treatment & Random cause treatment & $\Delta$ CATE \\
\hline ATE & 0.113 & - & - & - \\
Backdoor logistic & 0.152 & 0.0005 & 0.151 & +0.039 \\
IPW & 0.212 & -0.0009 & 0.212 & +0.099 \\
DML & 0.146 & 0.0014 & 0.146 & +0.033 \\
\hline
\end{tabular}

It can be seen that in all causal models, accounting for confounders further strengthened the effect of the survey location. That is to say that inference made on a traveler's existing mode choice is an effective method of predicting future trip, especially if demographic variables and confounders are causally treated. This effect changed by an upwards of near $10 \%$ as seen in the inverse propensity weighting study. Furthermore, all estimates pass refutations, as their placebo experiments reduced the effect of the study variable to near zero, and the random common cause treatment did not change the effect estimation significantly.

A more brief causal study can be done to study the effect of considering traveler's gender as an instrumental variable affecting the correlation between high income earners and the inclination to choose Swissmetro. Under a basic ATE, the effect of a traveler's status as high income increased the Swissmetro choice inclination by just 0.029, with a standard error of 0.019. Even when using a more involved method, such as IPW, the effect is returned as 0.044. However, when the estimation accounts for the traveler's gender as an instrumental variable as previously described, high income earner status increase the Swissmetro choice inclination by 0.120 .

\section{Conclusions}

To further examine the effectiveness of using a traveler's existing mode choice to predict future inclinations, a series of causal inference studies were carried out. After adjusting for 
confounders, a survey respondent's likelihood to choose an innovative trip mode provided that they have previously traveled on a train is increased, and the assumption to use previous trip mode choice as a predicting variable can be strengthened if causal variables are properly treated. Furthermore, it is demonstrated that a full analysis of instrumental variables can strengthen existing models that use income status as a trip mode predictor. 


\section{References}

Gianluca Antonini, Carmine Gioia, and Emma Frejinger. Swissmetro: description of the data. 2007.

A Bauranov, S Parks, X Jiang, J Rakas, and MC González. Quantifying the resilience of the us domestic aviation network during the covid-19 pandemic. front. Built Environ, 7: $642295,2021$.

Michel Bierlaire, Kay Axhausen, and Georg Abay. The acceptance of modal innovation: The case of swissmetro. In Swiss Transport Research Conference, number CONF, 2001.

Timothy Brathwaite and Joan L Walker. Causal inference in travel demand modeling (and the lack thereof). Journal of choice modelling, 26:1-18, 2018.

Hilmi Berk Celikoglu. Application of radial basis function and generalized regression neural networks in non-linear utility function specification for travel mode choice modelling. Mathematical and Computer Modelling, 44(7-8):640-658, 2006.

Josh Everts and Xuan Jiang. Making sense of electrical vehicle discussions using sentiment analysis on closely related news and user comments. arXiv preprint arXiv:2112.1232\%, 2021 .

Julian Hagenauer and Marco Helbich. A comparative study of machine learning classifiers for modeling travel mode choice. Expert Systems with Applications, 78:273-282, 2017.

Hichem Omrani. Predicting travel mode of individuals by machine learning. Transportation Research Procedia, 10:840-849, 2015. 